\title{
Participación e incidencia de las OSC: una apuesta para la construcción de políticas públicas en Pereira ${ }^{1}$
}

\author{
Participation and incidence The CSOs: a bet for the construction of public \\ policies in Pereira
}

Joaquín Andrés Gallego Marín²

Cesar Augusto Castaño ${ }^{3}$

Erika María Bedoya ${ }^{4}$

\begin{abstract}
Gallego M, Joaquín. Castaño, Cesar. Bedoya, Erika miradas $\mathrm{N}^{\circ} 1$ - 2018 ISSN digital $\mathrm{N}^{\circ}$ 2539-3812 Págs 125 - 143

Recepción: Agosto 14 de 2017

Aprobación: Abril 11 de 2018

Publicación: Junio 29 de 2018
\end{abstract}

\section{Resumen}

En el contexto de Pereira -Risaralda- 2011 a 2016 se analiza la incidencia de las Organizaciones de La Sociedad Civil (OSC) en la formulación e implementación de Políticas Públicas, entendiendo la incidencia como una acción que permite desarrollos discursivos deliberativas que ponen en interacción las dinámicas del mundo de la vida en el ámbito operativo de OSC entre el poder comunicativo, el poder administrativo y el poder social en la interlocución e interacción discursiva para el diseño de políticas públicas. Para el análisis de ésta interacción se parte de la política deliberativa de Jurgen Habermas, operacionalizado mediante la metodología de las 4C's como: cooperación, confrontación complementariedad y cooptación, en tres estadios: 1. la participación e incidencia de las OSC para la formulación; 2 en toma de decisiones, y 3. en ejecución de las políticas públicas: cultura de la legalidad y atención de víctimas. Se ultima que se

\footnotetext{
1 Artículo producto de la investigación: Incidencia de las organizaciones de la sociedad civil en el diseño y ejecución de políticas públicas en Pereira en los años 2012 a 2016. El proyecto se formuló para participar en la convocatoria interna del año 2016 de proyectos de investigación financiados por la Universidad Libre Seccional Pereira, Grupo de investigación en Derecho Estado y Sociedad.

2 Abogado; licenciado en Etnoeducación y Desarrollo Comunitario; magíster en Comunicación Educativa y candidato a doctor en derecho. Docente investigador de la Universidad Libre, seccional Pereira. joaquina.gallegom@unilibre.edu.co 3 Licenciado en Etnoeducación y Desarrollo Comunitario; magíster en Comunicación Educativa. Docente investigador de la Universidad Libre, seccional Pereira. cesara.castañog@unilibre.edu.co

4 Licenciada en Etnoeducación y Desarrollo Comunitario; magíster en Comunicación Educativa; suficiencia investigadora en el Doctorado en Paz Conflicto y Democracia; estudiante de Doctorado Interinstitucional en Educación. Docente investigadora de la Universidad Libre, seccional Pereira. erikam.bedoyah@unilibre.edu.co
} 
provee amplios aspectos de marginalidad concreta en lo que respecta a las OSC, entendidas como sujetos de incorporación activa y de gestación deliberativa de la política pública y sus componentes de desarrollo.

Palabras claves: políticas públicas, deliberación, participación, incidencia.

\section{Abstract}

In the context of Pereira -Risaralda- 2011 to 2016, the impact of Civil Society Organizations (CSOs) on the formulation and implementation of Public Politics is analyzed, understanding incidence as an action that allows for deliberative discursive developments that put in interaction the dynamics of the world of life in the operational field of OSC between communicative power, administrative power and social power in the interlocution and discursive interaction for the design of public politics. The analysis of this interaction is based on the deliberative politic of Jurgen Habermas, operationalized through the 4C's methodology as: cooperation, confrontation, complementarity and cooptation, in three stages: 1. the participation and incidence of OSCs for the formulation; 2 in decision making, and 3 . in execution of public politics: culture of legality and victim assistance. It is finalized that it provides broad aspects of concrete marginality with regard to OSC's, understood as subjects of active incorporation and deliberative gestation of public politics and its development components.

Keywords: public policies, deliberation, participation, incidence.

\section{Introducción}

Los procesos de construcción de las políticas públicas son del interés de todos los ciudadanos, organizados y no organizados, por cuanto definen la inversión de los recursos en acciones que mejoren la calidad de vida de todos. La constitución de 1991 abrió la posibilidad de que estas políticas fueran construidas de manera participativa. Desde esta perspectiva, el ejercicio de investigación postulado desde el proyecto Incidencia de las organizaciones de la sociedad civil (OSC) en la formulación y ejecución de la política pública en la ciudad de Pereira 2012-2016 pretende observar y reflexionar conjuntamente la teoría y el hecho social de la participación de las OSC en el contexto del municipio de Pereira, y su incidencia en la formulación y ejecución de las políticas públicas.

Este observar propuesto en la investigación requirió de especial atención ya que a través de este ejercicio es como se puede ejercer y posibilitar la participación de la sociedad en la esfera pública y en la agenda municipal. Sin embargo, más allá de definir si existe o no incidencia de las OSC en la agenda y las políticas públicas de la ciudad de Pereira, el proyecto se interesó por conocer los procesos de constitución y desarrollo de capacidades de deliberación de las OSC, los impactos de su participación en los asuntos relacionados con su propio desarrollo, y la atención que obtienen por parte de los tomadores de decisiones. Del mismo modo, se interesó por la forma como se despliega la incidencia (vía legal o ilegítima) a fin de diagnosticar los alcances de la movilización social en el diseño o ejecución de una determinada política pública. Con ello, se propende por contribuir en la construcción de los indicadores de inclusión social en la deliberación de los asuntos públicos. 
Hacer participación política supone la construcción de capacidades institucionales que pueden ser potenciadas a partir estrategias de formación y apropiación social de los conocimientos en las prácticas participativas, dirigidas no solo hacia la intervención en políticas públicas, sino a la construcción de ciudadanía y al establecimiento de nuevas relaciones con el Estado.

De esta manera, el estudio de la actividad de las OSC y el análisis de sus procesos de constitución y construcción de capacidades institucionales e incidencia en políticas públicas demanda el reconocimiento de experiencias concretas en el contexto de intervención desde escenarios participativos. Así las cosas, la pregunta que orienta esta investigación es: ¿En qué sentido la concepción de política deliberativa de Habermas complementa y ensancha el modo como los actores de las OSC inciden en la formulación y ejecución de políticas públicas en el municipio de Pereira, 2012-2016?

La incidencia es un concepto que puede definirse como la influencia o efecto que tiene una cosa sobre otra (Granados, 1994). Desde otra perspectiva, Craig Jenkins (1995) define la incidencia como los intentos de grupos, organizaciones y actores para influir sobre las decisiones de elites institucionales, en todos sus niveles de acción, en función de intereses colectivos o de grupos subrepresentados, incorporando en esta última categoría al público en general. Así, cuando se alude a la incidencia, se advierte una relación con la interpretación procesual de las políticas públicas en la que es importante reconocer, analizar y valorar en qué medida las acciones de incidencia se vinculan con una de las fases analíticas de la política pública y cuáles son las demandas que se generan para la activación institucional de las organizaciones.

El análisis sobre la incidencia supone el estudio de las diferentes fases de la política involucrada que refieren a la construcción del problema público, a la incorporación del problema en la agenda, a la formulación de una política y a la implementación y evaluación de la política público como respuesta a dicho problema. Asimismo, el análisis advierte sobre el estudio de las estrategias y los mecanismos de influencia y movilización utilizados por las OSC, que se traducen en repertorios de acciones conformes con sus iniciativas, intereses y propósitos en un campo particular de incidencia (social, político, económico, entre otros) y en una fase de la política sobre la que actúa.

Es en el contexto de la formulación de la política pública en el que se considera conveniente generar procesos interpretativos desde la órbita teórica de la acción comunicativa propuesta por Jürgen Habermas, desde la configuración del sentido de la participación ciudadana en los procesos políticos del Estado. Es desde esta discusión que se incorpora el referente de la política deliberativa fundada en la acción comunicativa, para complementar y ensanchar el modo como los actores de las OSC comprenden, participan e inciden en la implementación de las políticas públicas en el municipio de Pereira, en el marco constitucional de una formación social participativa. Profundizar la incidencia de la participación en el proceso de construcción de ciudadanía participativa presupone, además de participar en la formulación de las políticas, instituir una situación de 
diálogo abierto entre funcionarios de la administración municipal, actores de las OSC y académicos.

El desarrollo metodológico de esta investigación se desplegó desde la investigación cualitativa. Con un enfoque hermenéutico, que permitió pensar y reflexionar la realidad social desde un ejercicio discursivo, se identificó en los textos la comprensión del sentido de la noción de participación que está en la base del mandato constitucional y legal, para profundizar la democracia participativa y la interpretación del sentido de la deliberación democrática en Habermas. A partir de la revisión de los lineamientos de las políticas de cultura de la legalidad y de atención a víctimas, en contraste con el paradigma de una deliberación democrática - siguiendo procedimientos en derecho-, se determinó la naturaleza y el alcance de las acciones de incidencia de las OSC en el sistema político y administrativo de la gestión pública municipal de Pereira. Desde estos productos se precisó en qué sentido la concepción de política deliberativa de Habermas complementa y ensancha el modo como los actores de las OSC comprenden, participan e inciden en la formulación y ejecución de las políticas públicas en el municipio de Pereira.

Con el objetivo metodológico de analizar la participación de las OSC, inicialmente se identifican los acuerdos y documentos que soportan la formulación y diseño de las políticas públicas de cultura de la legalidad y de atención a víctimas. Para ello se establecen categorías teóricas que permiten analizar los documentos de política recopilados. El análisis se desarrolla a partir de la metodología de las $4 \mathrm{C}$, toda vez que permite revisar la participación desde
4 categorías teórico-prácticas. El proceso estuvo acompañado por el desarrollo de tres estadios que permitieron observar la dinámica de los actores sociales y del Estado en la concertación de una agenda temática, discusión, toma de decisiones e implementación de las políticas públicas.

En ese sentido, la propuesta metodológica desarrollada para el análisis de la incidencia de las OSC desde la política deliberativa de Habermas se convierte en un insumo metodológico pertinente para la operatividad de políticas públicas desde las administraciones municipales y las OSC. De esta manera, se constituye en uno de los resultados más significativos de esta investigación.

\section{La deliberación desde la óptica habermasiana para estudiar la política pública en el contexto de Pereira}

Abordar las políticas públicas implica comprender que son polivalentes, tanto en su concepción como en su definición. Al respecto, Vargas (1995) señala que se pueden encontrar de 20 a 30 definiciones diferentes de lo que son las políticas públicas, y como más, no existe una disciplina teórica particular que se encargue de su estudio. Sin embargo, en aras maximizar las posibilidades que pueden albergar, es posible conceptualizar las políticas públicas desde una posición sociocrítica en cuanto posibilidad de ser un espacio de razonar en público dentro de la dinámica del Estado.

No obstante, en el contexto de la incidencia de las OSC en el diseño y ejecución de políticas pública, su comprensión parte desde la orientación conceptual elaborada por la política deliberativa propuesta por 
Habermas (2005), como «la pieza nuclear del proceso democrático» (p. 372).

La forma de concebir la política deliberativa debe considerar «la política como un escenario de procesos de poder y la analizan desde el punto de vista de discusiones y enfrentamientos estratégicos por intereses, o de efectos de control y regulación de carácter sistémico» (Habermas, 2005, p. 363), siendo la primera concepción -el poder- la apuesta desde la deliberación como medio para operar el sistema político, y segundo -analizancomo procesos políticos que surgen desde la facticidad social que son observables. En consecuencia, la deliberación conllevaría a consenso que se forma desde el discurso para generar acciones basadas en el entendimiento comunicativo.

En sí, la deliberación está ligada a la posibilidad de que los individuos, no solo desde la esfera de la individualidad, sino también desde la sociabilidad - formada a través de su voluntad colectiva-, presenten buenas razones para respaldar las pretensiones de validez de las acciones. A propósito, Habermas (2005) entiende la deliberación como:

(...) una red de discursos y formas de negociación que tiene por fin posibilitar la solución racional de cuestiones pragmáticas, morales $\mathrm{y}$ éticas, es decir, justo de esos problemas estancados de una integración funcional, moral y ética de la sociedad, que por la razón que sea ha fracasado en algún otro nivel (p. 398).
Esta noción de deliberación se entiende como consenso de fondo para la toma de decisiones a las que se llega por acuerdos de entendimiento racionales planteados a partir los discursos emanados desde la esfera pública, es una perspectiva de deliberación que dista de los mecanismos de imposición entre los sujetos, el ciudadano y el poder administrativo, al ponerla en el contexto de la elaboración del procedimiento político. Así pues, esta forma de entender la deliberación tiene la peculiaridad de estar ligada a los procesos democráticos desarrollados por los ciudadanos desde el presupuesto de que existen desacuerdos entre éstos y el poder administrativo, por los intereses que cada uno tiene.

El planteamiento de la deliberación es un procedimiento político del ciudadano en cuanto actor político, inculcado desde la propia cultura — el mundo de la vida — «en que éste ha crecido y está» (Habermas, 2005, p. 372) para la toma de decisiones desde los entendimientos discursivos, siendo así una peculiar forma de construir la opinión y la voluntad política de los ciudadanos a partir de sus preferencias e intereses individuales como conflictos morales. Por ende, como plantea Habermas, la política deliberativa se configura mediante la base de la teoría del discurso, conformada por lo que él denomina la acción comunicativa. Con ella es posible el análisis de las opiniones y acciones que construyen los sujetos mediante la racionalidad producida desde el mundo de la vida (cultural, social, personal), donde se generan interacciones que pueden conllevar el cuestionamiento de saberes racionales en la sociedad, a construcciones e interacciones simbólicas en torno a la realización del mundo de la vida. Para Habermas (1998), «Las manifestaciones simbólicas de sujeto 
capaces de lenguaje y de acción sólo pueden ser identificadas mediante descripciones que hagan referencia a las orientaciones de acción (y a las posibles razones) de un actor» (p. 85), que, según el autor, se dan bajo las presuposiciones de que la persona parte desde estructuras simbólicas en torno a la realización del mundo de la vida mediante operaciones comunicativas:

\section{(...) al actuar comunicativa- mente los sujetos se entienden siempre en el horizonte de un mundo de la vida. Su mundo de la vida está formado de convicciones de fondo, más o menos difusas, pero siempre aproblemáticas. El mundo de la vida, en tanto que trasfon- do, es la fuente de donde se obtienen las definiciones de la situación que los implicados presuponen como aproblemá- ticas (p. 104).}

Lo que Habermas plantea conlleva que se desarrollen imperativos metodológicos en que los actores pasen a tomar posturas frente a las pretensiones de validez universal desde los saberes desde el mundo de la vida. En consecuencia, la acción comunicativa se comprendería como la interacción entre sujetos capaces de comunicarse desde la dimensión pragmática del lenguaje para conformar relaciones comunicativas y dialógicas de forma intersubjetiva. Por ende, el procedimiento para poder desarrollar ello es el discurso que se genera de las relaciones sociales.

Entonces, desde la perspectiva de Habermas, resulta que el discurso es un concepto procedimental de condición pragmática, ideal para la deliberación y toma de decisiones. Por ello, Habermas (2005) lo considera como «un procedimiento democrático» (p. 372) de la opinión y la voluntad política, que ha sido institucionalizada mediante la opinión publica elaborada informalmente por el ciudadano. De allí que Habermas indique que el discurso:

(...) establece una conexión
interna entre las considera-
ciones pragmáticas, los com-
promisos, los discursos de
autoentendimiento y los dis-
cursos relativos a justicia y
fundamentan la presunción
de que bajo las condiciones
de un suficiente suministro
de información relativa a los
problemas de que se trate y de
una elaboración de esa infor-
mación, ajustada a la realidad
de esos problemas, se consi-
guen resultados racionales,
o respectivamente resultados
[rectos] (p. 372).

Este concepto de discurso encierra la atribución de representar los procesos de entendimiento que se efectúan a través del procedimiento democrático conforme a la autodeterminación del ciudadano, pues es finalmente él quien debe decidir, de forma pública, cuando se dan los desacuerdos entre los individuos, o entre ellos y los sistemas.

En el discurso reposa en la capacidad del individuo para argumentar y desarrollar la acción orientadas hacia el entendimiento , entre la relación de la actividad ciudadana y la actividad estatal, teniendo como eje 
principal la opinión y voluntad política del ciudadano como agente racional en virtud de sus propios intereses hacia la legitimidad normativa. La forma de reflexionar la política pública desde la política deliberativa exige la elaboración de consensos acerca de lo se entiende por verdadero y correcto desde una racionalidad que le exige al ciudadano autonomía y madurez en los procesos democráticos, formación democrática de la opinión y voluntad política conformada por consensos sujetos a una decisión colectiva. Según Habermas (2005), «la política deliberativa obtiene su fuerza legitimadora de la estructura discursiva de una formación de la opinión y voluntad que sólo puede cumplir su función socio integradora gracias a la expectativa de calidad racional de sus resultados» (p. 381). Estos aspectos de la política deliberativa, en su trasfondo, demandan que el ciudadano sea un actor colectivo, de madurez ética, de alto nivel práctico, y que refleje el todo autoreflexivo para la interacción mediante el debate público como el lugar donde el individuo se construye como «un actor colectivo que refleja el todo reflexionado sobre él y que actúa por él» (p. 375).

Por lo tanto, las reclamaciones en favor o en contra de tales decisiones tienen que ser justificadas frente a estas personas en términos que, bajo la reflexión, sean capaces de aceptar. El aspecto reflexivo es crítico porque las preferencias pueden transformarse en el proceso de deliberación que surge por la práctica de autodeterminación del ciudadano en relación con el poder administrativo o las élites, mediante acciones comunicativas formadas por rectos criterios de valoración ético-normativa que el ciudadano y la administración asumen, pues estos deliberadores son capaces de cambiar sus juicios, preferencias y opiniones durante el curso de sus interacciones, lo que implica persuasión en lugar de coerción, manipulación o engaño.

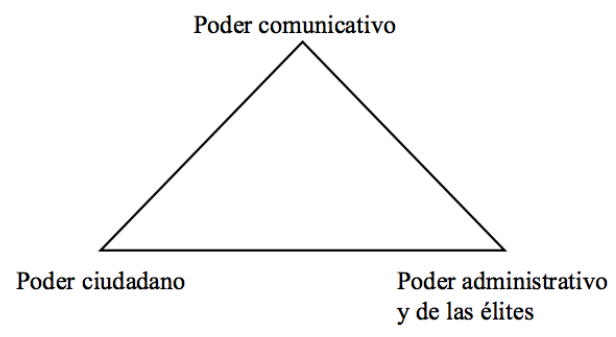

piupia.

Como bien lo advierte la noción de deliberación que Habermas (2005) propone, para respetar las reglas de juego democrático y generar el procedimiento demandado por la política deliberativa con miras a la producción legítima de normas, necesariamente se requiere contar con unos «ciudadanos racionales» (p. 371) De ello se sigue, la necesaria consideración de comprender con Habermas que la organización política de la sociedad demanda de altos grados de rectitud del ciudadano cuando contrae compromisos sociales para llegar, de forma justa, a los arreglos entre intereses. Estos Arreglos no necesariamente se dan por cooperación, pero si pueden provenir de la negociación de intereses, consensuados a partir de entendimientos ético- políticos en la relación social en la cual se desarrolla la política, "la sociedad es de por sí sociedad política, societas civilis" (pág. 373). Pero la rectitud que Habermas reclama se aboca a las demandas de los ciudadanos racionales, donde la política deliberativa entreteje las 
negociaciones de intereses, en un contexto concreto de acción, en una democracia abierta al debate en la esfera de la opinión pública, de allí que Habermas (2005) observe que "[la] política deliberativa constituye una pieza nuclear en el proceso democrático" (p. 372), y en consecuencia, el orden regulativo entre lo que es poder administrativo y el poder social.

En el trasfondo, la política deliberativa es una forma de hacer procedente la soberanía popular y de retroalimentar el sistema político, donde la toma de decisiones es colectiva y vinculante y donde los individuos deben aceptar las decisiones, toda vez que su desarrollo se concreta en los espacios públicos políticos o mediante procedimientos democráticos, y porque son producto del entendimiento, de formas $\operatorname{argumentativas}^{5}$ que justifican la opinión.

De todo ello, se puede considerar que el proceder deliberativo es consensual y no

5 Desde la presentación que Habermas hace de este concepto, en su libro Teoría de la Acción comunicativa, la argumentación es un ejercicio de interacción comunicativa cuya finalidad es conformar consensos mediante acuerdos que institucionalizan lo justo y lo correcto. Para Habermas (1998), «los argumentos asumen papeles distintos desarrollando en el discurso un grado distinto de fuerza vinculante» (p. 324). Por ello, la forma de construcción de los argumentos la posesiona la teoría del discurso:

Sólo esa teoría del discurso podría señalar explícitamente en qué consiste la unidad de la argumentación, y articular qué es lo que podemos querer decir con la expresión «racionalidad procedimental» tras haber quedado disueltos por la crítica todos los conceptos sustanciales de razón (p. 324). También, y observando lo que Habermas plantea en Facticidad y Validez, la argumentación se muestra como el medio que permite que los participantes del diálogo justifiquen sus pretensiones de validez ante el público que no está condicionado, y tiene la capacidad de entender el sentido de los argumentos:

En este sentido la acción comunicativa remite a un argumento en el que los participantes justifiquen sus pretensiones de validez ante un auditorio idealmente ampliado. Los participantes en la argumentación parten de la suposición idealizadora de una comunidad de comunicación delimitada en el espacio social y el tiempo histórico (Habermas, 2005, p. 400). agregacionista, como indica Adela Cortina (2009), en especial porque «la deliberación tiene la capacidad para transformar las preferencias iniciales y para modular una voluntad autointeresada» (p. 180), ubicando una concepción procedimental más allá del objetivismo empleado por el comunitarismo para constituir las legitimidades de las normas jurídicas y políticas. Asimismo, como indica Cortina, la forma más eficiente de distinguir la función deliberativa de la agregacionista es cuando esta pretende «[dar] por supuesto que las preferencias y los intereses se forman en privado, que es preciso partir de ello como un hecho dado, y no contempla la posibilidad de transformarlo, sino únicamente de agregarlos» (p. 180), y por lo cual, el sujeto agregacionsita:

\section{(...) tienen una noción men- guada de racionalidad, que} no permite superar paradojas como la de la imposibilidad de Arrow ${ }^{6}$. Por el contrario, la racionalidad humana viene impregnada por los valores de los contextos concretos y por motivaciones que no se reducen al autointerés (p. 180).

Consecuentemente, teniendo en cuenta a Richardson, citado por Cortina (2009), se puede comprender la forma procedimental en que el proceso deliberativo que entraña la condición habermasiana, siendo un poco más explícito cuando establece cuatro etapas en el proceso deliberativo:

6 La paradoja de Arrow (o teorema de la imposibilidad de la democracia) de Kenneth Arrow, premio Nobel de Economía en 1972, demuestra que no es posible diseñar reglas para la toma de decisiones sociales o políticas que obedezcan a un criterios conjunto de criterios razonables. Arrow, K. J. (2012). Social choice and individual values. Yale university press. Nota que no pertenece al original 
Participación e incidencia de las OSC: una apuesta para la construcción de políticas públicas en Pereira

(...) la exposición de los términos del desacuerdo y las propuestas de posible solución, la argumentación que avale tales propuestas, un acuerdo informal en el que los participantes se comprometen a asumir la parte que les corresponde y a actuar conjuntamente, y la decisión oficial (p. 180).

Asimismo, los mecanismos para llegar a la decisión pueden ser tanto los que indica Richardson: el voto o la representación, como las políticas públicas, en donde también es posible encontrar un punto de vista común sobre cómo los ciudadanos toman públicamente decisiones desde desacuerdos.

Entonces, el planteamiento de la democracia a esta instancia de pensarse mediante la política deliberativa estaría en la necesidad de conformación mediante la racional y directa vinculación del ciudadano con el que se conforma la opinión y la voluntad política reguladas por procedimientos. La propuesta de la política deliberativa sería entonces el medio para llegar a la democracia deliberativa con la que se cristalizaría el modelo de democracia radical $^{7}$ propuesto por Habermas. En concordancia con lo indicado por Cortina (2009):

(...) la democracia es el gobierno del pueblo, los

7 Esta concepción propuesta por Habermas sería un medio de respuesta más efectiva, racional, y sobre todo, dialógica de los ciudadanos con la administración pública para exigir cambio de relación en la conformación de la práctica política en el Estado. ciudadanos deben ejercer su autonomía política, no sólo eligiendo representantes que deben responder de sus decisiones ante el pueblo, sino también tomando parte activa en esas decisiones a través de la deliberación (p. 181).

Cabe señalar también que, desde la idea de política deliberativa planteada por Habermas, la democracia se construye mediante la formación de la voluntad política del ciudadano, voluntad que conducen a decisiones colectivas mediante razones sin coerción. Así lo explica Dryzek (2000): «democratic legitimacy came to be seen in terms of the ability or opportunity to participate in effective deliberation on the part of those subject to collective decisions» (p. 119). Así entendida, el centro de estructuración de la deliberación está en la forma y el proceso en que se edifica, es el componente importante de la democracia, es un concepto de orden procedimental de ésta, con la que hay un cambio de perspectiva del papel de la sociedad como sociedad política.

En ese sentido se descentraliza la capacidad totalizante de la sociedad centrada en el Estado y se pasa a configuraciones de prácticas de autodeterminación política de los ciudadanos como comunidad consciente de sí misma, y en la cual se ejecutan autooperaciones mediante las voluntades colectivas de los propios ciudadanos en donde el concepto de democracia se conforma desde la autoorganización. Así, para Habermas (2005), «la democracia viene a significar auto organización política de la sociedad en conjunto» (p. 373).

Metodología de estudio de la política 


\section{pública en el contexto de Pereira desde el procedimiento de la deliberación}

La perspectiva metodológica empleada en esta investigación es hermenéutica. Los esfuerzos investigativos se centraron en la comprensión de la noción de participación que aparece en la base del mandato constitucional y legal con el objeto de profundizar la democracia participativa y la interpretación del sentido de la deliberación democrática en Habermas. A partir de la reconstrucción de las percepciones de los actores intervinientes en relación de contraste con el paradigma de una deliberación democrática, siguiendo procedimientos en derecho, se determinó la naturaleza, el alcance y la incidencia de las acciones de las OSC en el sistema político y administrativo de la gestión pública municipal de Pereira. En particular, el trabajo centró su mirada en los procesos de construcción e implementación de las políticas públicas, pues es en estos escenarios donde se observa, según Habermas (1998), esa «red de discusiones y formas de negociación que tienen por fin posibilitar la solución racional de cuestiones pragmáticas, morales y éticas, es decir, justo de esos problemas estancados de una integración funcional, moral y ética de la sociedad» (p. 398)

En concordancia con lo anterior, se retoma parte de la propuesta metodológica de Adil Najam (2000): «The Four C's of Government Third Sector-Government Relations $»^{8}$, la cual nos permite identificar la incidencia de las OSC en la solución racional de esas cuestiones pragmáticas, morales y éticas a través de las políticas públicas.
El modelo de las $4 \mathrm{C}$ analiza el interés estratégico y la preferencia política heterogénea entre actores sociales los cuales varían en función de las metas y los medios de unos y otros, estableciendo cuatro posibles escenarios estratégicos entre el gobierno y los actores sociales. Para nuestro caso (las OSC), los escenarios son la cooperación, la confrontación, la complementariedad y la cooptación (Figura 2).

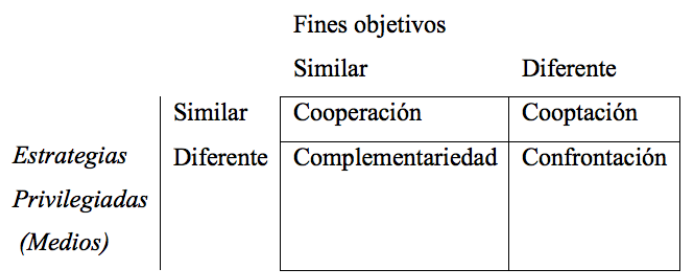

Figura 2. Las cuatro $\mathrm{C}$ de $\mathrm{ONG}$-relaciones gubernamentales. Fuente: adaptado de Najam (2000, p. 383).

- Cooperación: este escenario se caracteriza por la búsqueda de fines con medios similares mediante la coproducción y colaboración entre el gobierno y las OSC en la medida que estas cinco condiciones se den: pluralismo institucional, normas compartidas, coordinación de actividades, objetivos comunes y comunicación abierta.

- Confrontación: es el escenario contrario a la cooperación: la búsqueda de fines y objetivos contrarios o de extremos con medios diferentes. En este caso no hay puntos de encuentro. La divergencia puede actuar en dos vías: a) como control coercitivo por el gobierno; b) como desafío y oposición de las OSC. En esta categoría las partes actúan resistiéndose una a la otra.

- Complementariedad: en este escenario el gobierno y las OSC comparten 
fines y tienen objetivos similares; sin embargo, difieren en los medios o estrategias para su obtención. En este caso los fines pueden llegar a ser más complemento que los medios.

\section{- Cooptación: en este escenario} tanto el gobierno como las OSC tienen diferentes fines pero convergen en las estrategias; tanto el gobierno como las OSC pueden intentar cambiar los fines del otro, convenciéndolo de que los fines perseguidos por uno son un subconjunto de los fines del otro.

\section{Los estadios de la deliberación}

La metodología de las $4 \mathrm{C}$ planteada por Najamseubica enlosescenarios dediscusión y deliberación y toma de decisiones. Sin embargo, para la construcción y ejecución de la política pública de la ciudad de Pereira por lo menos es necesario establecer cómo se constituyen esos escenarios, cómo se establecen los temas de la agenda pública, cómo se construye el escenario de discusión de los problemas, necesidades y temas de interés de las OSC, el gobierno y la ciudadanía en general, y cómo se logra la concertación y ejecución de las posibles soluciones enmarcadas o reflejadas en las políticas públicas.

En este sentido, resulta necesario recordar que la Constitución Política de Colombia de 1991 consagra en el artículo 1 que «Colombia es un Estado social de derecho, organizado en forma de República unitaria, descentralizada, con autonomía de sus entidades territoriales, democrática, participativa y pluralista», circunstancia que implica un nuevo orden político-territorial desde la autonomía de los territorios que les otorga la facultad a los actores sociales de tomar sus propias decisiones, generando procesos de interacción con el Estado para la construcción de las políticas públicas que orientaran el desarrollo del territorio. Es por ello que para el estudio de la incidencia de las OSC en la construcción de la política pública se plantea desde el desarrollo de los siguientes tres estadios.

El primero se establece desde el mismo momento en que surge la necesidad temática por parte de las OSC o la organización del Estado (desde la administración central o descentralizada) que tiene relación directa con las necesidades sentidas por la población de la entidad territorial o del propio Estado como aparato administrador. Este estadio se puede considerar como el inicio del establecimiento de los temas que serán la base para las posibles agendas de trabajo, lo que nos pone en un escenario de entender el origen o la historia de la política pública.

El segundo es el escenario convocado por la administración pública. Converge allí la sociedad civil a partir de sus intereses y se priorizan los temas de interés público para su discusión, concertación y construcción de la política pública. Este estadio se puede considerar como el de construcción, legitimación y legalización en el orden de la entidad territorial.

El tercero es la implementación, la operacionalización, el monitoreo y la evaluación de la política pública en el contexto territorial en relación con las OSC, la sociedad no organizada, los individuos y agremiaciones públicas y privadas, locales y no locales, que pueden ser cooperantes o veedores del ente territorial que implemente la política pública. 
Sin embargo, metodológicamente, ante estos tres estadios, cada uno de ellos tiene implícita la producción y transformación de información entre las OSC (como: mundos hermanos, USAUD, REDDIS, red de organización de víctimas y KAIROS) y los actores de la administración pública, pues tanto unos como otros inciden en la política pública.

En el primer estadio se dan las acciones iniciales (ex/ante) para la posible elaboración de una política pública. Allí se establecen los siguientes actos:

- Primer acto. Convocatoria de actividades de acción con el propósito de concitar la necesidad de una política pública en una línea temática: en este acto los actores sociales realizan convocatorias, acciones sociales de hecho y acciones programadas con el objetivo de sensibilizar o hacer evidente una problemática social o tema de importancia para la sociedad.

- Segundo acto. Actividades de encuentro preliminar de reunión conjunta de los actores: una vez establecidos los temas de interés común para los actores sociales y los entes gubernamentales se desarrollan encuentros previos entre las partes, para conocer las agendas individuales de las partes, donde se evidencias las lecturas de contexto, los intereses, objetivos y metas individuales de las partes, que se evidencias en las convocatorias actas de dichos encuentros.

\section{- Tercer acto. Agendas individuales} de los actores: la construcción de la agenda de discusión para la construcción de la política pública es el producto de los encuentros preliminares.

En estos actos se mueven y ejercen deliberaciones preliminares a partir de intereses particulares o colectivos que se observan desde los registros de información secundaria y primaria, permitiendo dar cuenta de los discursos de intereses y pretensiones de los actores de las OSC y del gobierno hacia la meta de la construcción de la política pública.

Para generar una estructuración y análisis de información de lo acontecido entre los diferentes actores se hace una síntesis de la información de primer y segundo orden que arroja datos del cómo, cuándo y mediante qué estrategias losactorespropusierontemas de interés público que desencadenaron la creación de políticas públicas, bien sea desde las necesidades sociales o intereses particulares de los actores sociales o desde la institucionalidad, es decir, entes locales, regional y nacionales.

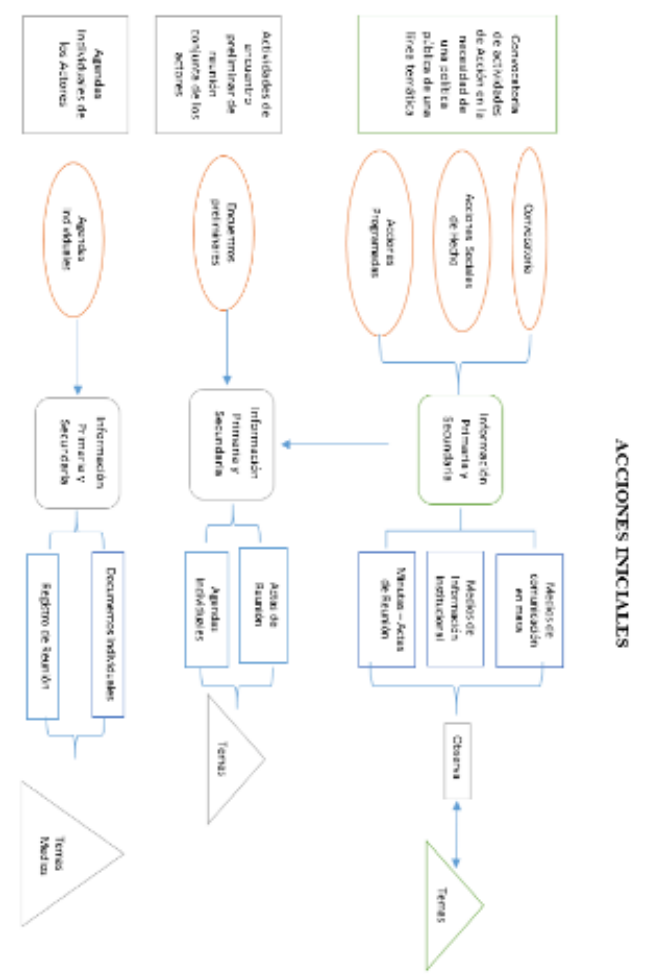

Figura 3. Estadio inicial. Fuente: Elaboración propia 
En el segundo estadio se siguen desarrollando acciones de continuidad (ex/ ante) y se establecen los siguientes actos:

\section{- Primer acto. Agenda común de} trabajo: se elabora desde las concertaciones de agendas individuales para la agenda común. Esta agenda es registrable a través de información de segundo y primer orden mediante el conjunto de actas de registro de las sesiones donde se generaron las discusiones para la identificación y determinación de problemas que llegan a llamar la atención, la identificación de alternativas ante los problemas identificados, la evaluación de opciones y la selección de opciones de política pública.

- Segundo acto. Formulación de la política púbica: se pasa de la agenda común de trabajo a los diferentes momentos de encuentro para la definición de problemas, la identificación de los medios posibles para alternativas de solución a los problemas, la selección de opciones para proponer alternativas de políticas públicas; también se definen las metas, los procesos de discusión, la concertación, y las participaciones e innovaciones que se concretan en el acuerdo municipal que formaliza las acciones desarrolladas ex/ ante. El medio de información adecuado a este acto se puede ubicar en las actas de discusión y concertación entre los actores $\mathrm{y}$, asimismo, el acuerdo municipal en donde se legalizan las legitimidades construidas en el consenso.

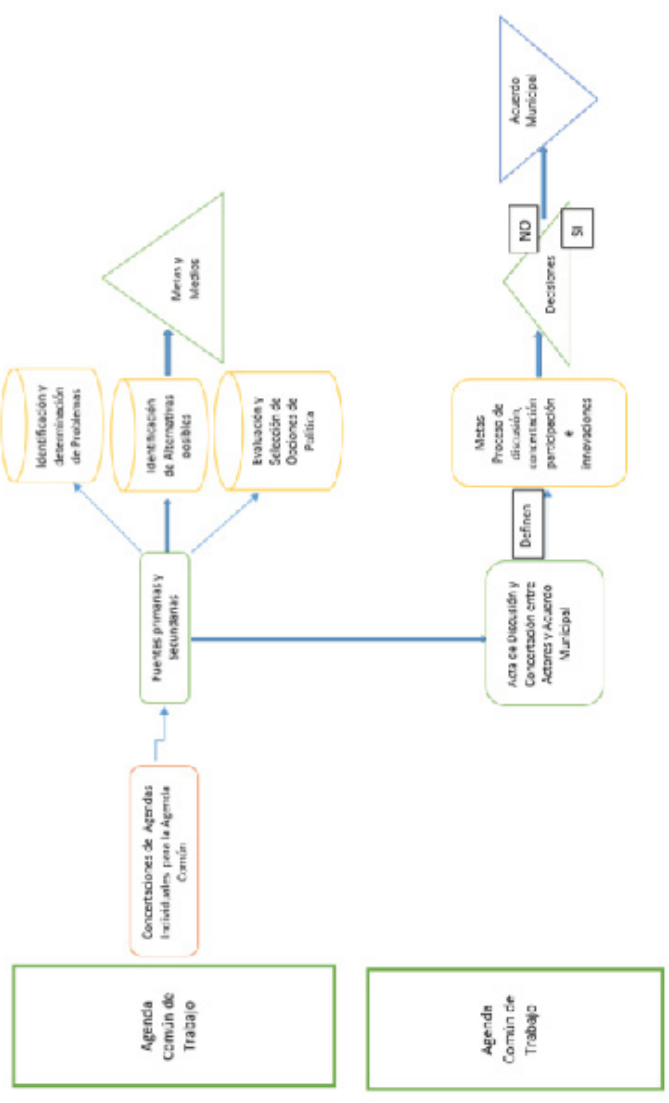

Figura 4. Acciones de continuidad. Fuente: Elaboración propia

El tercer estadio es un momento ex post del proceso, cuando ante la existencia de un documento normativo, se generan acciones de implementación, monitoreo o seguimiento, y evaluación de la política pública. En este momento es posible observar la capacidad administrativa de las entidades territoriales para llevar a cabo la intencionalidad propuesta en el documento de política pública. Los actos desarrollados en este estadio son:

- Implementación: la acción de implementación de la política pública es el momento cuando las entidades territoriales, mediante sus unidades 
administrativas, estructuran (planes, programas y proyectos) e instrumentalizan procedimientos administrativos (recursos humanos y provisión de servicios) para el cumplimiento del objetivo estimado en esa política. En tal sentido, el desarrollo de los procesos de estructuración e instrumentalización son objeto de seguimiento mediante información de primer y segundo orden proporcionada por los agentes, funcionarios y actores, tanto de las OSC como de la administración pública, quienes se vinculan o interfieren en los actos de estructuración e implementación de la política pública. Otras fuentes de información de segundo orden son los planes de desarrollo municipal, y los programas y proyectos institucionales e interinstitucionales formulados por las secretarías y despachos de la administración pública o las OSC.

- Monitoreo o seguimiento: las acciones de monitoreo o seguimiento a la política pública se plantean desde el origen de la política hasta su término o tiempo de finalización. Necesariamente toda política pública cuenta con un tiempo estimado de ejecución. Es en ese periodo de vigencia de la política cuando se supone que desde las acciones de implementación se desarrollan todos los componentes que la han estructurado a fin de satisfacer la necesidad diagnosticada, para lo cual sirvió la conformación del documento de política pública. Posteriormente se entra a evaluar el resultado de las acciones de implementación (monitoreo o seguimiento de las acciones de implementación). Las acciones y resultados de la implementación de la política pública son objeto de monitoreo o seguimiento por dos vías: a) por la información de primer y segundo orden producida por los actores de las OSC y la administración pública. Parte de esta información es revisable mediante los informes de seguimiento de políticas públicas que hace la administración pública por cada año de vigencia del plan de desarrollo y a través de posibles informes construidos por los actores de las OSC intervinientes en la política pública, y b) por desde las experiencias de los actores intervinientes.

- Evaluación: la acción de evaluación es el momento que permite hacer valoraciones y juicios finales sobre la política pública. En esta etapa se determina cuáles son los cumplimientos -o formas de generar cumplimiento- de lo estimado en la política pública. El trasfondo de la evaluación es procurar la eficacia del aparato organizacional del Estado para la gestión de los recursos, las capacidades y la diversidad de iniciativas, y asegurar la idoneidad de los acuerdos e intervenciones de abordaje de los problemas, como resultado de la aplicación de las políticas públicas. La manera de observar el resultado de la política pública es mediante los informes finales o de resultados registrados por la administración pública, contando con el complemento de la información primaria suministrada por expertos evaluadores o auditores del proceso. 


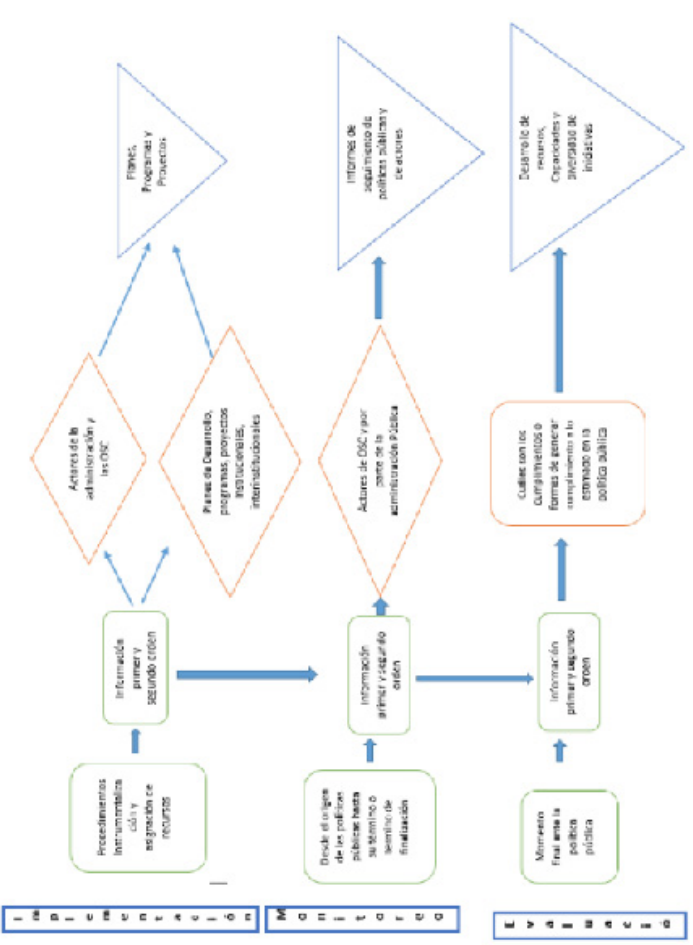

Figura 4. Acciones ex post. Fuente: Elaboración propia

Ahora bien, vale recordar que el objetivo de la investigación se centra en determinar la incidencia de las OSC en la construcción y ejecución de las políticas públicas de la ciudad de Pereira, mediante la deliberación con el ente territorial y que la metodología de las $4 C^{\prime}$ 's nos permite observar el resultado de esa deliberación; asimismo, también es pertinente subrayar que esta investigación se centró en los estadios dos (priorización de los temas de interés público para su discusión, concertación y construcción de la política pública) y tres (que es cuando se implementa, operacionaliza, monitorea y evalúa de la política pública).

Con este fin se analizaron los documentos que contienen las políticas públicas, los acuerdos del concejo municipal y el documento oficial que da cuenta de las políticas públicas. En ellos se determina cómo identifican a los ciudadanos y a las OSC, hacia quien se dirige la política pública y cómo se concibe. También se realizó una caracterización de las OSC que participaron en la construcción y ejecución de las políticas públicas del Plan de Atención a Víctimas (PAV) y cultura de la legalidad mediante una matriz de caracterización y entrevistas. Luego se efectuó un análisis de las memorias de las discusiones realizadas en el marco de la construcción de las políticas públicas con el fin de identificar los objetivos y medios de los diferentes actores e identificar qué escenarios se dieron: de cooperación, de contradicción, de complementariedad o de captación. Finalmente se analizaron las matrices de seguimiento y cumplimiento de las políticas públicas para identificar la incidencia y participación de las OSC en el proceso de implementación.

\section{Avances del estudio de la política pública en el contexto de Pereira desde la concepción de la incidencia}

La incidencia pertenece al hacer de la participación de las OSC en el diseño de las políticas públicas, ya que estas hacen parte activa de los desarrollos de la administración municipal y de la política en sí, que, en palabras de Habermas, tienen un carácter de regulación sistémico que denotando ejercicios de poder guiados por las discusiones y los intereses de los participantes.

En ese sentido, las prácticas políticas deben aportan a la participación efectiva de todos los actores, que en alguna medida se ve reflejada por las acciones discusivas que llevan consigo los intereses de todos los actores y que legitiman la fuerza de 
la democracia como un hacer constitutivo de su dinámica. Ello permite desarrollar regulaciones de fuerza entre poderes, que en palabras Habermas (1998), refuerzan la «capacidad que tienen de imponerse intereses superiores que pueden ser perseguidos de forma más o menos racional, el poder político podría concebirse como una forma abstraída de poder social articulada de forma estable sobe el poder administrativo» (p. 364).

Es así como las OSC deberían expresar su legitimidad, medida a través del reconocimiento e incidencia de su participación en el diseño de las políticas públicas; sin embargo, en este estudio se pudo determinar que esta participación pierde legitimidad cuando la estructura política no incluye desde los determinantes iniciales de la construcción de las políticas una participación de dichas organizaciones, en que las acciones de intercambio y cooperación se convierten en actividades de recepción de información, más en una lógica de confrontación entre administración pública y OSC conllevo a una formulación de la política pública e implementación de ésta por iniciativas no discutidas con el poder administrativo de Pereira.

De esta manera, las políticas públicas revisadas en el proceso investigativo (cultura de la legalidad y atención a víctimas PAV) denotan ejercicios de construcción muy particulares, pero que parecen constantes en los procesos de construcción de esas políticas. En el caso de la política de la cultura de la legalidad, ella se articula con base en los lineamientos del plan de desarrollo de Pereira (2008-2011). Con este fin, inicialmente la alcaldía constituyó un subprograma para desarrollar durante dicho periodo, pasando luego a constituirse como política pública en el año de 2010 , propuesta que parte, según documento técnico en la cultura de la legalidad:

\section{(...) del trabajo realizado por} parte de la administración municipal y las entidades del sector privado, las organizaciones sin ánimo de lucro, las iglesias católicas y cristianas, las autoridades de policía, las agremiaciones artísticas, el sector educativo y los medios de comunicación en coordinación con la Agencia Estadounidense para el Desarrollo Internacional (USAID), con el apoyo del Centro Nacional de Información Estratégica (NSIC) (Alcaldía de Pereira Secretaria de Planeación Municipal, 2010, p. 11 ).

Respondiendo a esta misma dinámica desde el hacer, la política de atención a víctimas PAV nace desde la idea de desarrollar la ley 1448 de 2011, la que a nivel local se resuelve con el acuerdo 65 de 2011 del Concejo Municipal de Pereira. El objetivo del acuerdo es:

Fortalecer la capacidad institucional del municipio Pereira a través de las Secretarias de Despacho e instituciones descentralizadas, de forma tal que estas puedan establecer e implementar acciones efectivas para la prevención, atención y protección de las personas en situación de desplazamiento en el municipio de 
Pereira, permitiendo niveles progresivos de restitución de los Derechos que les fueron vulnerados (Concejo Municipal de Pereira, 2011; p. 3).

Es así como se encuentran puntos de reflexión en torno a la incidencia de las OSC en el diseño y ejercicio de políticas públicas, ya que estas responden a lineamientos a nivel nacional que luego son adaptadas a nivel local a partir de programas institucionalizados desde el ejercicio administrativo municipal. Empero, es allí donde se pierde el sentido deliberativo de la incidencia de las OSC, toda vez que el discurso no resulta atravesado por espacios que permitan la aplicación de una metodología participativa que supere el sentido informativo y de socialización, arriesgándose a producir escenarios que permitan la aplicación de las 4 C's como un instrumento que legitima el ejercicio democrático.

El municipio de Pereira cuenta con instrumentos que permiten realizar el seguimiento de la ejecución de las políticas públicas y permiten revisar, en términos de indicadores, las metas alcanzadas y los recursos asignados para la efectiva aplicación de los programas que contienen las políticas públicas. Allí, de una u otra manera, las OSC hacen presencia, ya que hacen operativas o bajan a la realidad las posibilidades que ofrece la política pública; sin embargo, este ejercicio no está mediado por un actuar de incidencia que permita el equilibrio de los grupos de interés, ni su interacción, ni ejercicios de legitimación, denotando un ejercicio de incidencias no representativo o democrático.
En la potencialización de la incidencia como un ejercicio democrático en términos reconstructivos debe en palabras de Habermas (2005) contener fragmentos que permitan establecer relaciones al interior de un sistema político articulado e incluyente, conformando un carácter de validez al estado de derecho y al ejercicio democrático, cuando las acciones de incidencia son permitidas desde escenarios de construcción de política, mediada por las acciones discursivas, permiten la deliberación y entender la importancia de la construcción conjunta en las decisiones de país.

La teoría del discurso se constituye como un procedimiento ideal para la deliberación. Este procedimiento democrático establece una conexión interna entre las consideraciones pragmáticas, los compromisos, el discurso de autoentendimiento y los discursos referentes a la justicia e información cercana a la realidad, los derechos humanos universales eticidad. Asimismo, esta teoría del discurso no depende solo de una capacidad de acción colectiva de la ciudadanía, sino también de la institucionalización de los correspondientes procedimientos y presupuesto comunicativos, así como la interacción de deliberaciones institucionalizadas con opiniones públicas desarrolladas informalmente.

\section{Conclusiones}

Como conclusión se puede indicar que las políticas públicas, en el contexto de la ciudad de Pereira (en especial, de las políticas públicas en cultura de la legalidad, plan de Atención a Víctimas), posee amplios aspectos de marginalidad concreta en lo que respecta a las OSC, entendidas 
como sujetos de incorporación activa y de gestación deliberativa de la política y sus componentes de desarrollo.

A partir de lo encontrado en los documentos y de la información recopilada y analizada en torno a las políticas públicas (cultura de la legalidad y Plan de Atención a Víctimas), se encuentra que están direccionadas o parametrizadas por intereses del orden nacional, lo cual implica un proceso de construcción de arriba hacia abajo mas no de abajo hacia arriba, desconfigurando una relación discursiva horizontal que termina la deliberación conjunta y donde adquiere mayor relevancia el poder discursivo.

En los documentos de acuerdos de las políticas públicas de atención a víctimas y cultura de la legalidad, en el ejercicio discursivo no se visibiliza al sujetociudadano como agente activo deliberativo que incide en la formulación y ejecución de las políticas públicas, aunque se les invita a los procesos de socialización no son visibles desde el año de conformación de ambas políticas.

Los procesos de interlocución donde convergen los poderes administrativos, políticos y sociales para la construcción de programas y proyectos para el desarrollo de las políticas o su estado de evaluación, se presentan las actividades e indicadores de cumplimiento bajo la opinión y voluntad regulada por procedimientos del poder administrativo y no por los procesos que involucran aportes de las OSC.

Losindividuos deciden, prediceny proponen acciones a partir de sus experiencias de vida social y política desde el contexto de lo público, en que deliberan y consensúan procesos normativos que los acercan a sus realidades, y posibles soluciones, sin embargo, estas deliberaciones y decisiones no son evidentes en los acuerdos ni en las políticas publicas

\section{Referencias}

Alcaldía de Pereira - Secretaria de Planeación Municipal, 2010, Política Publica en Cultura de la Legalidad.

Concejo de Pereira. Acuerdo 65 de 2011. (29 de diciembre de 2011). Por el cual se adopta la política pública Plan Integral Único para la Población Víctima de Desplazamiento Forzado pro (sic) la violencia reubicada en el municipio de Pereira como instrumento de la política pública para garantizar el goce efectivo de los derechos 2011-2015. Disponible en: https:// www.pagegear.co/es/dominios/concejopereira/ upload/acuerdos/AC 65_2011.pdf

Constitución Política de Colombia. (1991). Disponible en: http://www.alcaldiabogota.gov. co/sisjur/normas/Norma1.jsp?i=4125

Cortina, A. (2009). La política deliberativa de Jürgen Habermas: virtualidades y límites. Revista de Estudios Políticos (nueva época). (144), abril-junio, pp. 169-193. Disponible en: $\quad$ https://dialnet.unirioja.es/descarga/ articulo/3014369.pdf.

Dryzek, J. (2000). Deliberative Democracy and Beyond: Liberals, Critics, Contestations. Oxford: Oxford University Press.

Grandos, J. A. T. (1994). Incidencia: concepto, terminología y análisis dimensional. Med clin (Barcelona) pp. 140 - 142

Habermas, J. (1998). Teoría de la acción comunicativa (Tomo 1: racionalidad de la acción y racionalidad social). Madrid, España: Taurus.

. (2005). Facticidad y validez. Sobre el derecho y el Estado democrático de derecho en términos de la teoría discursiva. Madrid, España: Trotta. 
Jenkins J. C. (1995) Social movements, political representation, and the state: An agenda and comparative framework. The politics of social protest: comparatives on states and social movements, 14 - 35

Naja, A. (2000). The Four C's of Government Third Sector-Government Relations. Noriprofit Management \& Leadership, 10(4), pp. 375496.

Salazar V, C. (1995). Las políticas públicas: nueva perspectiva de análisis. Ciencia politica, (38), pp. 59-78. 